\title{
1. Understanding creativity, innovation and entrepreneurship in healthcare
}

\section{QUESTIONS}

What do you understand by the term creativity? What role does creativity play in your healthcare organization? Why is creativity important? What is innovation and what does it mean in the context of healthcare? What is the link between creativity and innovation? Is creativity important for innovation to emerge and develop within healthcare organizations? Why? What is entrepreneurship in healthcare? In terms of creativity, innovation and entrepreneurship, what is the future for healthcare?

\section{INTRODUCTION}

Creativity, innovation and entrepreneurship research has been developed over decades across many disciplines and is of major importance to the field of healthcare. Given the global healthcare challenges its importance has increased significantly among researchers and practitioners. Globally, healthcare is experiencing major challenges as a result of, for example, aging populations, rising costs, regulations, long waiting lists, inequitable care, inaccessible care, medical errors, and challenges in staff recruitment and retention, all of which impact the timing and response to patient diagnosis, quality and safety. Current approaches are short term, focusing on a single issue with no overarching solution to fully address the challenges. However, there are significant opportunities to address these challenges through creativity, innovation and entrepreneurship.

The terms creativity and innovation are not always differentiated. There are, however, important differences between the two concepts that need to be acknowledged. Effective creativity results in the generation of novel and valued ideas. There is no innovation without creativity because creativity is the foundation on which innovation emerges, develops and grows. Creativity focuses on the generation of ideas, processes or concepts, while innovation focuses on the practical viability of those ideas, processes or concepts. Creativity can result in new inventions; however, innovation is the commercialization of such 
inventions, that is, putting the creative idea into action. To achieve this, healthcare organizations must utilize their resources and the creative abilities of their people. Creativity and innovation cannot happen without people who have core competencies, drive, motivation and passion to make a difference. It is the creativity among individuals that develops innovation; however, this must be supported and encouraged by appropriate leadership within the healthcare organization. From a healthcare organizational perspective, developing creativity in individuals throughout the organization will generate greater patient value. From an individual perspective, when individuals are creative they are more confident, motivated and feel part of the organization. This results in higher productivity among staff and potentially more innovative ideas and a more entrepreneurial mindset.

Entrepreneurship is a universal concept that can be applied to new ventures, small- to medium-size enterprises, large national and multi-national organizations, and public sector organizations including healthcare. Within healthcare, entrepreneurship requires strong commitment, perseverance and research to develop something novel that will generate patient value. The entrepreneurial spirit needs to be engendered and nurtured to successfully bring medical innovations to fruition.

The purpose of this chapter is to develop an understanding of creativity, innovation and entrepreneurship in healthcare organizations and examine how healthcare can prosper through creativity, innovation and entrepreneurship. The chapter provides an understanding of the meaning and importance of creativity, innovation and entrepreneurship to healthcare. Following this, the link between creativity, innovation and entrepreneurship is discussed. The chapter concludes by providing an overall framework for Leading Entrepreneurship and Innovation in Healthcare: A Global Perspective that will serve as the foundation for the structure of this book.

\section{CREATIVITY: MEANING AND IMPORTANCE IN HEALTHCARE}

\section{What is Creativity?}

Creativity is a core component of innovation and fundamental for the development and generation of innovative ideas. Creativity can be defined as an individual's ability to recognize and develop new ideas, processes or concepts in novel ways - thus creating something new, useful and valuable. In healthcare, creativity is originality that is realistic, viable and novel that will enhance healthcare in order to generate real patient value. Creativity is critical to healthcare to allow us to re-think the current approach, by looking at things in new ways and facilitating the generation of ideas and creative solutions to 
address the challenges faced by patients, healthcare professionals, stakeholders and society at large.

Creativity is not just a revolutionary changing product or service that comes from globally recognized innovators like Professor Alexander Fleming who discovered penicillin in 1928 and Dr. Raymond Damadian known as the "father of the MRI" (Magnetic Resonance Imaging) in 1969. Creativity in healthcare requires cognitive and non-cognitive skills, curiosity, intuitiveness, perseverance, passion and commitment. To generate patient value in healthcare, individuals need to utilize their creativity despite potential barriers within internal and external environments. Individual and team creativity requires the following:

- An open and objective mindset

- Perseverance and commitment to generate novel ideas

- Passion to generate patient value

- Moderate risk taking

- Resilience to overcome obstacles

- Ability to recognize existing ideas in other industries that could be effectively utilized within healthcare.

Creativity is demonstrated by innovative organizations in healthcare such as: One Medical (affordable and convenient primary care); Apple (mining data to build health apps); Alivecor (mobilizing heart monitoring); and GE Healthcare (reducing pain points at healthcare facilities). Furthermore, technological advancement and development is rapidly changing healthcare as a result of the significant developments in creativity and innovation, for example, artificial intelligence (AI), 3D printing, robotics, telemedicine, wearable monitors, stem cells, gene therapy, mobile device applications. Therefore, effective utilization of creative behaviors among individuals with diverse competencies can lead to successful innovations in healthcare.

\section{Why is Creativity Important?}

There is a continuous need to enhance and develop healthcare and generate greater patient value. Creativity is the important first step for innovation. Healthcare continues to be a highly complex sector with healthcare professionals required to provide more creative solutions in their care practices. Creativity that is supported and facilitated can encourage engagement from staff at all levels and show that their creative ideas generate patient value and are core factors in the success of the healthcare organization and the field of healthcare. Creativity is essential for the growth and development of healthcare staff. To achieve this growth, staff must be open to evaluating the gaps and limitations 
in the current system, and exploring new areas and ways to achieve desirable goals and objectives. In doing this, healthcare professionals need to manage the needs of a diverse group of patients in terms of, for example, age, current and pre-existing health conditions, lifestyle, and making creativity a fundamental element of their day-to-day work. Therefore it is critical that staff are given the time and support to engage in creativity to address these growing demands. The integration of creativity into patient care can generate significant benefits such as decreasing costs, increasing efficiency and effectiveness, ensuring greater utilization of resources, and improve quality and safety.

\section{Creative Techniques}

Creative techniques must be utilized in order to generate creative ideas and concepts. There are many techniques that can be used to generate them, including:

1. Brainstorming: 6 to 12 team members generate a number of ideas without any negative criticism and then evaluate each idea.

2. E-Brainstorming: a form of e-collaboration.

3. Brainwriting: a silent version of brainstorming where the generated ideas are recorded individually on a piece of paper and submitted anonymously to the group. The ideas are exchanged a number of times with each person building on the previously generated ideas.

4. Focus groups: individuals providing information in a structured format.

5. Free association: writing down a word or phrase related to the problem, followed by another and another, with the goal that each new word will add something new to the ongoing thought processes and thereby generate a chain of ideas, finishing with the emergence of a new product idea.

6. Mind mapping: allows an individual or team to generate numerous ideas by dividing each idea into many more detailed ideas.

7. Collection notebook method: individuals or teams consider the problem and potential solutions, recording ideas at least once but ideally three times daily. After a week, the best ideas are listed, as well as any suggestions.

8. Problem inventory analysis: a method for generating new ideas and opportunities by focusing on existing problems.

The value of these techniques depends on the individual's thinking process, problem-solving ability, and decision-making. The thinking process is usually better if participants have diverse perspectives, backgrounds, experiences, skills, and expertise; this avoids groupthink and enhances "out-of-the-box" thinking. The goal of creative techniques is to manage creativity in a more 
systematic way and improve the quality of the creative output, resulting in a high-quality concept or solution.

\section{INNOVATION: MEANING AND IMPORTANCE IN HEALTHCARE}

\section{What is Innovation?}

Innovation is a process that begins with an idea; proceeds with the development of an invention; and results in the development or enhancement of products, processes, services, or technological advancement as part of organizational/ industry innovativeness (Hisrich and Kearney, 2013). Innovation in healthcare begins with a new idea or concept leading to the development of a product, service, process, technology or delivery method with the ultimate goal of generating patient value and an enhanced healthcare system. The World Health Organization (WHO) recognizes that innovation in healthcare adds value in achieving greater efficiency, effectiveness, quality, sustainability, safety and/ or affordability (World Health Organization, 2016).

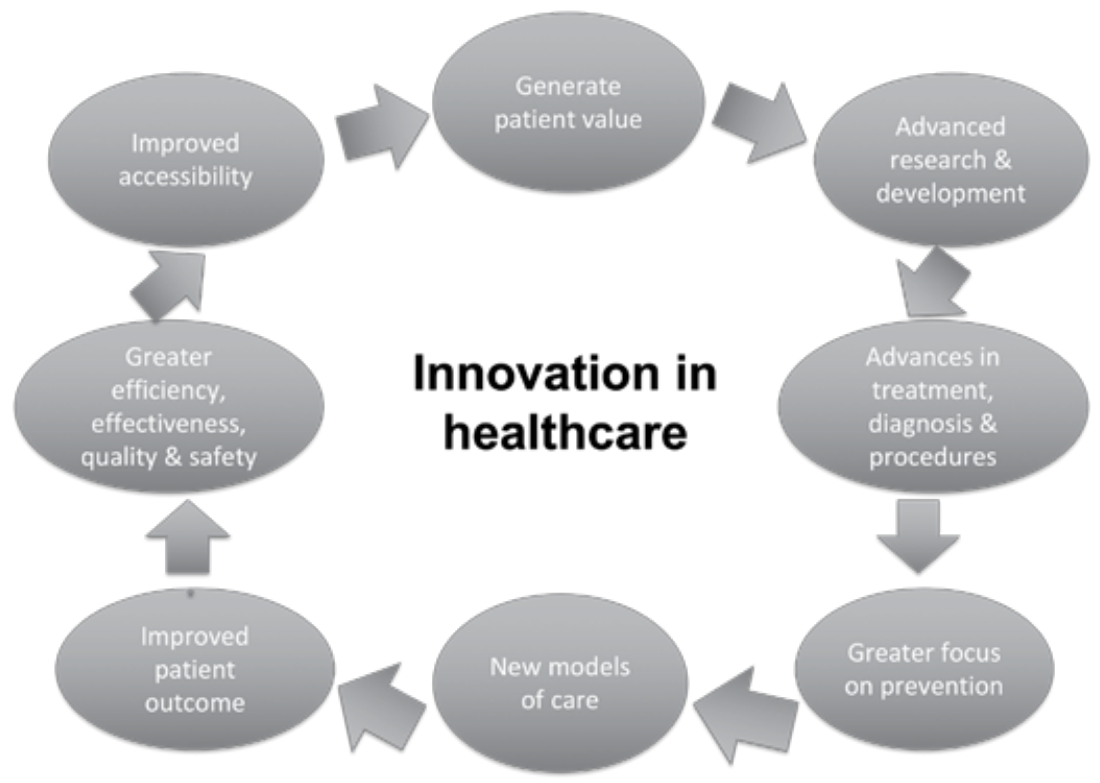

Figure 1.1 Value of innovation in healthcare 
The value of innovation in healthcare is recognized through, for example, the generation of patient value; advanced research and development (R\&D); advances in treatment, diagnosis and procedures; greater focus on prevention; new models of care that result in, for example, improved patient outcomes, greater efficiency, effectiveness, quality and safety; and improved accessibility for all patients (Figure 1.1).

Innovation in healthcare needs to continuously work toward addressing the unmet needs of patients who are at their most vulnerable. This also includes making existing innovations available to individuals that previously had no access to such treatment and care. This is particularly important in developing countries, because in order to improve access and equity and decrease costs there is a need to leverage existing technologies, for example mobile phone and genomics that are at the core of innovation in healthcare. Furthermore, innovation needs to focus on ways of prevention as well as cure for the well-being of all in society. While the medical profession focuses on early detection of specific illnesses that increases survival, there is a need to go a step further and identify ways individuals at particular risk of certain illnesses can prevent or reduce their risk.

Healthcare organizations in today's complex and dynamic market need to be innovative and support new creative ideas. Innovative healthcare organizations encourage and facilitate new ideas. They are creative with a willingness to take risks as they seek new improved processes, practices and technologies. The most successful innovative healthcare organizations are those that utilize knowledge gained from previous innovations within and outside their sector to develop future innovations. By reviewing innovations in other industries there is an opportunity for potential solutions to healthcare services that otherwise may not be recognized. For example, the rigidity of safety checks in aviation could be adopted in hospitals to work toward a reduction in medical errors. While innovation in healthcare requires new innovations for unmet and unsolved healthcare needs, it also needs novel approaches for addressing existing needs.

\section{Why is Innovation Important?}

The healthcare sector needs innovation. Healthcare providers, life science companies, health insurance companies and governments are facing increasing costs and pressures to generate patient value. Innovation is a core focus as healthcare organizations face unprecedented challenges. The continuous need for more innovation in healthcare has escalated as a result of the growth in demand due to aging populations, increasing prevalence of chronic diseases, an increase in unmet medical needs, more people living an unhealthy lifestyle, and an increase in patient demands for quality, safety and accessibility. 
Innovations in healthcare should improve patient care, enhance quality and safety, reduce harm and errors, increase accessibility, increase efficiency and effectiveness, eliminate waste, and lower costs. As a result of greater innovations in diagnostics and therapeutic options, healthcare has developed many innovations designed to enhance life expectancy and quality of life. Innovation in terms of new products, processes, services, technologies or delivery method is central to address these needs and achieve a more effective and sustainable healthcare system.

Technological innovations brought about by robotics, virtual reality (VR), automation, AI, 3D printing and drones have the potential to disrupt and transform healthcare. These technological advancements are used in some countries, yet others lag behind; for example, Ireland needs to recognize the opportunities such technological advancement can create and consider exploiting these technologies to transform the delivery of healthcare to patients, including more timely diagnosis, greater precision in surgery and improvement in the accuracy and administration of drugs. However, in time these rapidly developing technologies, such as AI, crowd-sourced health data, gene therapy, genomic sequencing, mobile phone applications, 3D printing, robotics, smart pills, stem cells, synthetic biology, telemedicine and wearable monitors, will continue to be further advanced and developed and will have a significant impact on the future of healthcare for patients, healthcare professionals, healthcare providers, payers, policymakers and all stakeholders.

The healthcare sector is changing as a result of, for example, innovations such as rapid growth of machine learning; genomics and precision medicine; digital technologies; a focus on patients at the center of their own care; and AI that can spot lung tumors more accurately than medical experts. These innovations are transforming medicine and create both opportunities to be recognized and achieved as well as challenges to address for the healthcare sector. The recognition of these opportunities and challenges is critical for the development of successful innovations to meet important unmet needs of patients, providers and payers.

\section{Characteristics of Innovation in Healthcare}

Innovation represents something new; this can result in a new product, process, service, technology, or delivery method. Whether innovation is a modification of an existing innovation or a transformational breakthrough offering something unique, it still results in a new way of doing and modifies the thought process to generate a new way of thinking about things. 
Innovation in healthcare needs to engage in the following:

1. Develop new products, services, processes, technologies or delivery methods in healthcare;

2. Develop new, more efficient and effective diagnostics, procedures, and treatment pathways;

3. Identify new approaches to promote health, prevent disease, and provide patient care and cures;

4. Extend patient care beyond existing methods so they are tailored for the specific setting, for example homecare.

Innovation in healthcare must deliver patient value by providing diagnostics and treatments that are timely, efficient and effective, with as little invasiveness and as few side-effects as possible in an approach that does not just meet but exceeds patients' needs, wants and expectations, and with dignity and respect. Innovations are not always planned, with some of the most successful being unplanned, for example penicillin. Therefore, the field of healthcare needs to be open to potential innovations beyond and in contrast to what they are aiming to achieve.

\section{CREATIVITY AND INNOVATION IN HEALTHCARE}

Creativity without innovation does not create any real value. Likewise, creative ideas are necessary to commence the innovation so it can develop and grow. Creativity and innovation do not just happen; it requires both general knowledge and field-specific knowledge, because creative individuals cannot know what is novel without an understanding of existing knowledge in any specific area. Within healthcare this also requires looking beyond the field of healthcare to discover how innovations in other areas may play a significant role in healthcare. We need to think differently if we want a different outcome. Creative people are always willing to challenge the way we think and not accept the status quo, because doing what is always done will achieve the same results. If those results are not favorable, we need to address this to develop creative ideas and implement innovations that achieve more desirable results and address current limitations within the system.

Healthcare leaders need to recognize the importance of creativity and innovation in their day-to-day operational activities and take action to foster this within their organizational setting. It is the most viable and effective solution to address the increasing challenges across every aspect of the healthcare system. This can only be achieved through strong leadership that supports and empowers staff to be creative and pursue innovation that will contribute to enhance healthcare services and generate patient value. To achieve this the 
leader needs to spend time listening to followers and patients to find out what goes wrong and then discover how to fix the problems as a team. They must empower staff to use their creativity to identify innovation(s) that will generate patient value and ensure the delivery of quality and safety while utilizing resources and decreasing waste that occurs within the healthcare delivery system. Creativity and innovation as a healthcare management goal can further enhance the organization of healthcare services and result in novel solutions to address challenges, such as increasing efficiency and effectiveness in communication and coordination across different departments, and ensuring appropriate processes for patient discharge that result in enhancing patient outcomes.

\section{Barriers to Creativity and Innovation in Healthcare}

Barriers to creativity and innovation in healthcare include:

- Regulation, rules, and policies. While it is necessary to enforce strict regulations and guidelines on healthcare innovations to ensure patients are always at the center of care and treatment, innovators must recognize and understand the regulations that affect their innovations from the outset, otherwise they can inhibit "out-of-the-box" thinking and slow down the innovation process.

- Bureaucratic environment. Rigidity, red tape, centralization and top-down communication can be typical of some healthcare organizations. These inhibit creativity and innovation becoming embedded within the organization.

- Culture of risk aversion. The healthcare sector can be risk adverse with primary concerns of accountability, quality and safety. Medical professionals are trained to determine the most proven method for treating disease, and to adopt that unless a more effective method was tried, tested and proven. This impedes creativity and innovation in finding and proving better ways of doing things.

- Pressured work environment. There is limited time to dedicate to creativity and innovation given highly stressed work environments and long working hours. Medical professionals are committed to the delivery of quality patient care, but the pressure to do more with limited resources can make it difficult to engage in creativity and implement innovations.

Healthcare is a highly dynamic complex sector and a healthcare delivery ecosystem can be under intense pressure as a result of increasing costs and patient needs. These challenges, combined with the complexity of the sector, make innovation in healthcare more complicated. To address these challenges 
and develop creativity and innovation in healthcare, these barriers must be overcome.

\section{Ways of Overcoming Barriers to Creativity and Innovation}

- Compliance and knowledge of regulations, rules and policies throughout the creative and innovative process.

- An organic organizational structure, decentralized decision-making and open channels of communication that facilitate creativity and innovation.

- A culture that encourages and supports creativity and innovation with a focus on delivering better healthcare.

- Utilization of resources for creativity and the implementation of innovation that will generate patient value.

\section{ENTREPRENEURSHIP: MEANING AND IMPORTANCE IN HEALTHCARE}

\section{What is Entrepreneurship?}

Within the literature there is no universally accepted definition of entrepreneurship. There are different forms of entrepreneurship, such as private sector entrepreneurship, public sector entrepreneurship, corporate entrepreneurship (also known as corporate venturing, organizational entrepreneurship, and intrapreneurship), governpreneurship, and social entrepreneurship. Entrepreneurs can be found in all professions, such as education, medicine, research, law, finance, architecture, engineering, technology, social work, and government. Entrepreneurs are recognized as individuals who are innovative and proactive, and who effectively manage risk. They have the ability to get things done despite the challenges or obstacles they may experience. Entrepreneurship in healthcare creates novel interventions, products, processes, services technologies or delivery methods that address health problems. There is a continued need for entrepreneurs and entrepreneurship within healthcare to bring about further needed innovations that will generate patient value.

The term entrepreneurship can be traced back almost three centuries with one of the earliest definitions from Richard Cantillion (written in the 1730s but not published until 1755), an economist, who refers to the entrepreneur as a rational decision-maker who assumes the risk and manages the organization. The classical contributors of the term entrepreneurship are recognized to be Cantillon, Say, Schumpeter, Knight and Kirzner. Some definitions focus on the creation of a new business, some on the exploration and exploitation of opportunities, while others focus on the generation of wealth and ownership, 
and the creation of value. Others focus on corporate entrepreneurship, which is a term used to describe entrepreneurial behavior within existing medium to large organizations including healthcare. However, as discussed by Drucker (1985):

The all but universal belief that large businesses do not and cannot innovate is not even a half-truth; rather, it is a misunderstanding (135). It takes special effort for the existing business to become entrepreneurial and innovative. ... The temptation in the existing business is always to feed yesterday and to starve tomorrow. It is, of course, a deadly temptation. The enterprise that does not innovate ages and declines. And in a period of rapid change such as the present, an entrepreneurial period, the decline will be fast (137).

The value of corporate entrepreneurship in healthcare is the degree to which it becomes part of the strategy to engage in entrepreneurship to generate patient value. While there is variation among the forms and professions, they do, however, incorporate similar concepts such as the exploration and exploitation of opportunities, creativity, innovation, risk taking, and generating value. A broad definition of entrepreneurship and corporate entrepreneurship "is the process of creativity and innovation by committing the necessary time and energy, taking responsibility for all the risks and uncertainties, and taking personal satisfaction (Hisrich and Kearney, 2013: 9). Understanding the importance of entrepreneurship in healthcare, the following definition recognizing critical factors is proposed:

Entrepreneurship in healthcare is a dynamic and challenging process of creativity and innovation that identifies and exploits previously unexploited opportunities. It requires energy, passion and commitment toward the creation and implementation of new ideas and creative solutions in light of the challenges and obstacles within healthcare. Essentially it requires the willingness to take calculated risks, formulate an effective team of experts with diverse competencies, ability to access and utilize resources, with the core goal to generate patient value.

Four key elements of this definition for healthcare are:

1. Entrepreneurship is a step-by-step ongoing process that can be applied to healthcare;

2. It exploits opportunities to address unmet healthcare needs;

3. It demonstrates passion and drive to pursue the opportunity despite the challenges healthcare professionals experience in their organizations;

4. It focuses on generating patient value and leading the world to better health.

In healthcare, entrepreneurship involves the process of bringing commercially viable innovations to the market or applying them within the organization, 
despite potential barriers. However, unlike other sectors, entrepreneurship in healthcare is more challenging as it is dealing with patients' lives. Therefore, all healthcare innovations need to be rigorously tried and tested, comply with all regulations, and demonstrate effectiveness and be recognized as being innovative. Entrepreneurship in healthcare needs to be driven by innovation while also being risk conscious; this requires high levels of creativity to be successful. Finding the balance in being innovative while operating in a risk-averse sector is challenging.

\section{Why is Entrepreneurship Important?}

There is a growing global importance for entrepreneurship in healthcare with the increasing needs of society to address more illnesses, diseases and conditions. The impact of an increase in chronic diseases places a major burden on a challenged healthcare system. Furthermore, the majority of countries are dealing with a shortage of doctors and medical professionals, and are experiencing challenges in the recruitment and retention of medical professionals. Staff shortages combined with healthcare system challenges create a major need for healthcare to promote entrepreneurial activities to drive innovation and generate patient value. The growth of entrepreneurship is disrupting the way healthcare is being practiced and is significantly and positively affecting the sector.

\section{LINKING CREATIVITY, INNOVATION AND ENTREPRENEURSHIP}

\section{Integration of Creativity, Innovation and Entrepreneurship to Healthcare}

How can patient value be created in healthcare through the integration of creativity, innovation and entrepreneurship? This requires looking outside of healthcare and reviewing the value generative approach of highly innovative and sucessful entrepreneurs and their organizations, such as Richard Branson (Virgin), Patrick Collison and John Collison (Stripe), Reid Hastings and Marc Randolph (Netflix), the late Steve Jobs (Apple) (whose legacy continues with Tim Cooks), Larry Page and Sergey Brin (Google), and Mark Zuckerberg (Facebook). They are highly creative, innovative and entrepreneurial, and they support and facilitate their staff to be the same. It is their competencies combined with curiosity, intuitiveness, perseverance, passion and commitment as well as a team to engender this passion for innovation that has resulted in this success. These competencies are needed in healthcare if we are to achieve the potential of the healthcare system and lead the world to better health. 
There is technological disruption globally within the field of healthcare and creativity; innovation and entrepreneurship are the catalysts. However, healthcare organizations find it challenging to incorporate the right strategies to initiate creative activity among their staff. To drive creativity, innovation and entrepreneurship within the sector, healthcare professionals must be empowered to utilize their creativity to identify innovative ways to deliver healthcare. The role of patients in the creative and innovative journey is fundamental as they are the key drivers of opportunity generation within healthcare organizations to generate patient value. Healthcare leaders and staff need to meet and engage with patients as part of the innovation process to gain greater knowledge and understanding of their specific experience.

\section{Benefits of Creativity, Innovation and Entrepreneurship}

Globally, healthcare professionals and providers are engaging in creativity, innovation and entrepreneurial activities driven by the desire to generate patient value. This has significantly contributed to healthcare over recent decades as a result of innovations such as advanced diagnostic and therapeutic options developed to improve life expectancy and quality of life.

The benefits of creativity, innovation and entrepreneurship are evident at three levels: individual, organizational and societal. From the individual perspective this instills strong passion, drive and motivation. At the organizational level, engaging in this process generates greater synergy and creates a positive and collaborative work environment. Hence, when an organization is more entrepreneurially oriented it creates the opportunity to develop new possibilities and ways of delivering that are characterized by innovation, risk-taking and proactivity. Within the field of healthcare, the link between individual and organizational goals can exceed the boundaries of the organization because of the "fit" between the altruistic nature of the organizational goals and the motivation and commitment healthcare professionals have to work to help others and benefit society (Kearney et al., 2020). For society this leads to better diagnostics, enhanced quality and safety, cost savings, and greater patient engagement and outcomes.

For continued benefits to the field of healthcare creativity, innovation and entrepreneurship need to accelerate further to develop where they are most needed, in areas such as prevention as well as cure, personalized care aligned to the patient's specific needs and genetic profile, and more technologically enhanced care models. This is required at individual and organizational level to benefit society and transform healthcare to have real-world impact and value. 


\section{Significance to Healthcare}

Patients are assuming greater responsibility for their health and well-being. Therefore the focus of healthcare is changing from managing illness to improving health and ensuring greater engagement in medical decisions. Through the advancement and development of digital technology there is an opportunity to bring more outpatient procedures out of overcrowded hospitals and back into the community. Patients do not have to physically attend a doctor's surgery when they are unwell as they can see a doctor through video conferencing from their own home. Additionally, technological innovations such as smart devices, smartwatches and medical apps facilitate doctors to remotely assess and monitor patients, thus increasing efficiency and decreasing surgery appointments.

Patients do not always need to go to hospital for a surgical procedure but can now have certain surgical procedures performed as an out-patient, for example a colonoscopy. Nursing homes are now one of a number of options for end-of-life care as home-care options and assisted living is providing more choices for those with palliative care needs.

Globally there is a transition toward personalized healthcare that empowers the patient. For example, TickerFit, an Irish company, developed a product used in cardiac rehabilitation that allows clinical teams to prescribe, educate and monitor a patient's recovery from a distance through a wearable device. This type of approach could result in taking patient care out of the hospital and increasing community care, thus reducing pressure on hospitals and offering more personalized treatment plans for patients. This is a timely and much needed approach to a pressured healthcare system.

\section{Helping to Save and Extend Lives}

Innovation in healthcare will continue to significantly increase life expectancy and quality of life, improve healthcare outcomes and reduce the cost of healthcare, leading the world to better health. Innovation is crucial to delivering high-quality, safe and affordable healthcare. The rapid advancement in digital technology and AI is revolutionizing healthcare with faster diagnosis and treatments, and potentially supports prevention rather than cure. For example, medical image diagnosis is critical to radiology due to the error rate (caused because radiological images include organs, bones and overlapping tissue, which makes it difficult for the radiologist to accurately identify problems), particularly at earlier stages of development. Additionally, technological advancement can reduce costs and increase efficiency and effectiveness through the development of more advanced, accurate and affordable diagnoses and treatments. 


\section{Leading the Way to the Future of Healthcare}

Healthcare organizations are leading great efforts to transform healthcare to make it more efficient, effective, accessible, affordable, convenient and patient-centered. Healthcare organizations must be innovative in terms of diagnostics, treatments and models of patient care. Like all industries the future is unpredictable but what is clear is the need to decrease the cost and increase the value of healthcare by delivering superior performance in terms of patient value through the creation of new innovative products, processes, services, technologies or delivery models, and appropriate strategic alliances. There is a change within the healthcare landscape therefore to manage this effectively; it is imperative to influence where it is going by harnessing innovation and entrepreneurship that will result in better patient care and lead healthcare into the future.

\section{A Framework for Leading Innovation and Entrepreneurship in Healthcare: A Global Perspective}

Leading innovation and entrepreneurship in healthcare has generated interest in recent years by academics, entrepreneurs, healthcare professionals, healthcare organizations and policymakers. While innovation is fundamental for organizational success it is one of the most difficult aspects of corporate activity to effectively manage and plan. When we reflect on the healthcare sector, this challenge is even more extensive due to the intense pressure on the healthcare market to "do more with fewer resources" and at the same time ensure the highest standards of quality, safety and patient care. Globally there is a pressing need for healthcare organizations to become more innovative and engage in entrepreneurial activities to stay ahead. In order to develop and grow an organization, and respond to the increasing and diverse needs of patients, leaders and healthcare professionals need to have innovation and entrepreneurship embedded in their organizational strategy. This requires the creation of an organizational strategy, structure, culture, rules, regulations, policies and procedures that encourages innovative behavior and rewards staff for such behavior. With increasing challenges facing healthcare there is a desperate need for the generation of new ideas and new innovative solutions to address deeply embedded problems on a global scale. Harnessing innovation, through the development of new products, processes, services, technologies or delivery models, will significantly contribute to better healthcare.

This book focuses on the important topic of leading innovation and entrepreneurship in healthcare from a global perspective. To be creative, engender innovation and facilitate an entrepreneurial spirit in healthcare requires knowledge of the process from idea generation to commercialization; additionally, 
knowledge on how to lead and develop an organization to emerge within this process is required. To guide the readers through this journey an integrative framework for leading innovation and entrepreneurship has been developed. As indicated in Figure 1.2, the framework has four major components:

1. Meaning and nature of innovation and entrepreneurship in healthcare;

2. Developing innovation and entrepreneurship in healthcare: a strategic perspective;

3. Leading innovation, entrepreneurship and design thinking in healthcare;

4. Making it all happen: a future-oriented mindset.

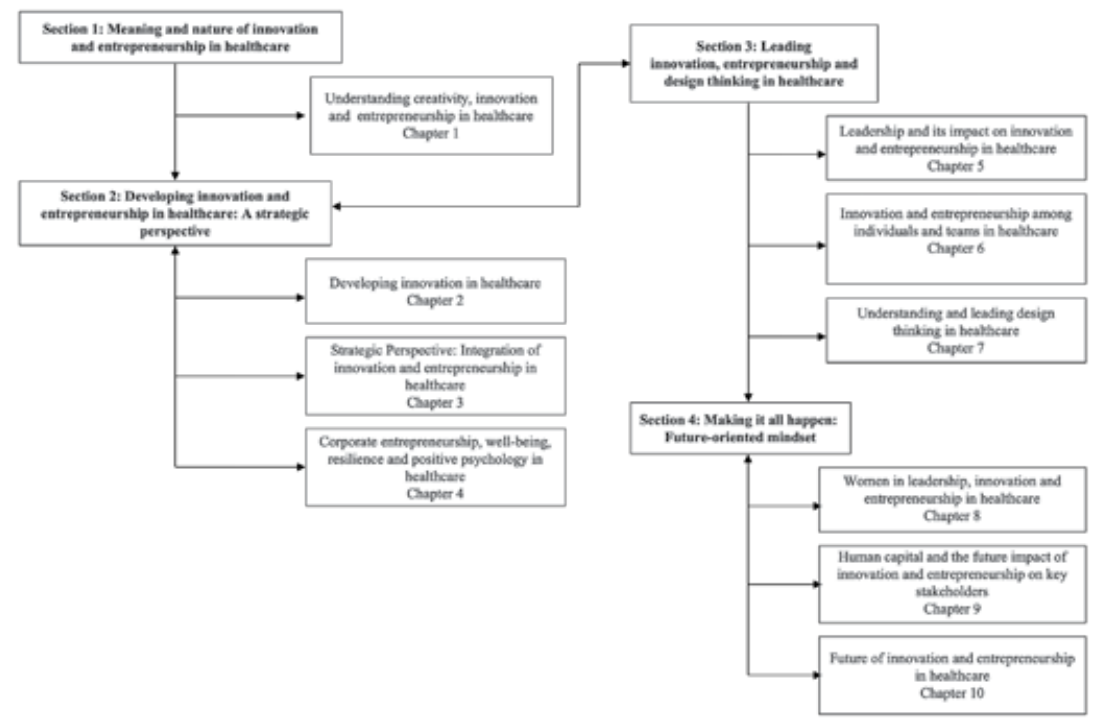

Figure 1.2 A framework for leading innovation and entrepreneurship in healthcare: a global perspective

Part I of this book, "Meaning and Nature of Innovation and Entrepreneurship in Healthcare," comprising this chapter, demonstrates an understanding of creativity, innovation and entrepreneurship in healthcare. It has provided the foundation for this book by giving an understanding of the meaning, nature, importance and links between creativity, innovation and entrepreneurship in leading the way to the future of healthcare.

Part II, "Developing Innovation and Entrepreneurship in Healthcare: A Strategic Perspective," consisting of Chapters 2 through 4, adopts a strategic focus on the advancement of innovation and entrepreneurship within 
a healthcare setting. Innovation is examined by investigating the forms, types and opportunities for innovation in healthcare. The steps in the process of innovation are examined and the value of innovation in healthcare presented. Innovation and entrepreneurship need to be effectively integrated into the organizational strategy. A system for monitoring, assessing and evaluating the external and internal environment, to ensure the right strategic innovative and entrepreneurial decisions are made and actions taken, is discussed with the objective of building a patient-centered innovative entrepreneurial strategy. An innovative and entrepreneurial healthcare organization is one that allows staff to recognize and act on their creative and innovative potential. Such an approach can enhance the well-being and resilience of healthcare professionals who are motivated in their innovative and entrepreneurial endeavors. Finally, this Part concludes with a discussion on corporate entrepreneurship, well-being and positive psychology in healthcare, and how leaders can create a climate that enhances staff well-being and resilience for now and the future of healthcare.

Part III, "Leading Innovation, Entrepreneurship and Design Thinking in Healthcare," consisting of Chapters 5 through 7, addresses the leadership of innovation, entrepreneurship and design thinking among individuals and teams in healthcare. The importance and styles of leadership are discussed along with engendering effective leadership for an innovative and entrepreneurial healthcare organization. The importance of effective communication at all levels in healthcare is examined. The behaviors associated with innovation and entrepreneurship require effective leadership that supports and motivates individual and team creativity, while ensuring the delivery of the highest standard of care. A discussion of the concept of design thinking and its importance for creative solutions and leadership support for healthcare challenges concludes this section.

Part IV, "Making it All Happen: Future-oriented Mindset," consisting of Chapters 8 through 10, focuses on innovation and entrepreneurship in healthcare in the future. Women in leadership, innovation and entrepreneurship in healthcare and the importance of inclusivity in healthcare organizations are discussed. Innovation and entrepreneurship does not happen without the right human capital that can drive and excel at future innovations in healthcare. Innovation and entrepreneurship have a different impact on a diverse group of stakeholders. Therefore, this requires a comprehensive understanding of the effects that innovation and entrepreneurship have on patients, clinicians and healthcare professionals, healthcare organizations, and government and other key stakeholders. Future innovations in medical devices need to be mindful of potential cybercrime vulnerability and ensure that security is built into such devices. An understanding of past and present innovations is required in order to develop and effectively lead innovations for the future of healthcare. The 
future of innovation and entrepreneurship in healthcare is upon us and together we can make a difference!

\section{SUMMARY}

The development of creativity, innovation and entrepreneurship across every aspect of the healthcare sector is the most viable solution to the global healthcare crisis, although this is a challenge given bureaucratic organizational structures, risk aversion and management teams who may resist such a transition. The driver of creativity, innovation and entrepreneurship in an organization is the responsibility of the CEO and senior management team who need to lead the way to the future of healthcare, that requires changing the status quo.

Innovation in healthcare needs to ensure more accurate, efficient and timely diagnoses and treatment plans, prevention as well as cure, patient engagement in the process of innovation, training and education, and research in innovation and commercialization, with the long-term focus on generating patient value. The benefits of such innovations will result in a more efficient and effective healthcare organization and enhanced medical solutions for addressing the needs of each patient.

More than ever, healthcare organizations globally must address the growing challenges and this requires leaders who can lead the world to better health by driving creative, innovative, and entrepreneurial behavior among all individuals responsible for delivering healthcare.

\section{REFERENCES}

Drucker, P.F. (1985). Innovation and Entrepreneurship: Practices and Principles (New York: Harper \& Row).

Hisrich, R.D. and Kearney, C. (2013). Managing Innovation and Entrepreneurship: A Global Perspective (Thousand Oaks, CA: SAGE Publications).

Kearney, C., Dunne, P.J. and Wales, W. (2020). Entrepreneurial orientation and burnout among healthcare professionals. Journal of Health Organization and Management 34(1), 16-22.

World Health Organization (2016). Innovation. Available at http://www.who.int/ topics/innovation/en/ (last accessed September 13, 2016).

\section{Suggested Reading}

Ciani, O., Armeni, P., Boscolo, P.R., Cavazza, M., Jommi, C. and Tarricone, R. (2016). De innovatione: the concept of innovation for medical technologies and its implications for healthcare policy-making. Health Policy and Technology 5, 47-64.

In this article, the authors undertake a systematic review of the academic literature to summarize definitions of innovation in relation to medical devices 
which they classified according to the source of innovation, to the degree of discontinuity introduced and to the impact associated to the technology. Additionally, they have been compared with definitions adopted for drugs by main healthcare reimbursement agencies.

Patterson, F. and Zibarras, L.D. (2017). Selecting for creativity and innovation potential: implications for practice in healthcare education. Advances in Health Science Education 22, 417-28. https://link.springer.com/article/10.1007/s10459-016-9731 -4 .

In this article, the authors explore the use of a trait-based measure of creativity and innovation potential, and evaluate its efficacy for use in selection for healthcare education. The authors use a sample of 188 postgraduate physicians applying for education and training in UK general practice. Their research clarifies the associations between personality, and creativity and innovation. In particular, their study highlights the importance of motivation in the creativity and innovation process.

Pillay, R. and Morris, M.H. (2015). Changing healthcare by changing the education of its leaders: an innovation competence model. Journal of Health Administration Education 33(3), 393-410.

In this article, the authors draw a distinction between traditional managerial competencies and innovation competencies, and argue that the latter help define the domain of innovation education. The authors employ a multistage Delphi methodology, and provide evidence of a core set of 19 innovation competencies. They also provide evidence for a more experiential and immersion-based approach to innovation education.

Raadabadi, M., Fayaz-Bakhsh, A., Nazari, A., Mousavi, S. M. and Fayaz-Bakhsh, M. (2014). Organizational entrepreneurship and administrators of hospitals: case study of Iran. Global Journal of Health Science 6(3), 249-55.

In this article, the authors examine the entrepreneurial activities within the hospitals affiliated to Tehran University of Medical Sciences, Iran. Using a questionnaire containing 29 items, their analysis showed that the majority of the managers agreed with all five areas of entrepreneurship, namely the existence of innovation and innovative behavior, flexibility, decision-making, a rewarding and encouraging system, and a management system supportive of personnel's new ideas. The authors assert that entrepreneurial activities in healthcare can be improved through providing a suitable environment, adjusting reward and encouragement systems, giving more authority to subordinates, promoting awareness and education, and mobilizing managers to attract appropriate opportunities for the organization. 
Ratten, V. (2015). Healthcare organisations' innovation management systems: implications for hospitals, primary care providers and community health practitioners. International Journal of Social Entrepreneurship and Innovation 3(4), 313-22.

In this article, the author discusses the role of innovation and entrepreneurship for healthcare organizations as a way of adapting to change. The author highlights the importance of creativity in healthcare organizations. Managerial implications for healthcare organizations highlight the evolution of hospitals, primary care providers and community practitioners in utilizing innovative and entrepreneurial techniques.

Weintraub, P. and McKee, M. (2019). Leadership for innovation in healthcare: an exploration. International Journal of Health Policy Management 8(3), 138-44.

This article is written for those seeking to foster innovation in the health sector. The authors offer a narrative synthesis approach of eight theories and concepts that have been empirically shown to support innovation through all phases of the innovation process. 